\title{
Effects of low-frequency ultrasound combined with microbubbles on benign prostate hyperplasia
}

\author{
Shao-ling Yang, MD, ${ }^{* *}$ Ke-qiang Tang, MD; ${ }^{+}$Wen-kun Bai, MD; ${ }^{*}$ E. Shen, MD; ${ }^{*}$ Yi-wen Zhao, MD; ${ }^{+}$ \\ Yan-duan Lin, MD; ${ }^{*}$ Shu-liang Nan, MD; ${ }^{*}$ Hu Bing, MD*
}

*Department of Ultrasound in Medicine, Shanghai Jiao Tong University Affiliated 6th People's Hospital, Shanghai Institute of Ultrasound in Medicine, Shanghai, PR China; 'Department of Ultrasound in Medicine, Branch Hospital in Fengxian of Shanghai 6th People's Hospital, Shanghai, PR China

Cite as: Can Urol Assoc J 2013;7(11-12):e681-6. http://dx.doi.org/10.5489/cuaj.354

Published online November 8, 2013.

\section{Abstract}

Introduction: Our objective is to assess the effects of low-frequency ultrasound combined with microbubbles on benign prostate hyperplasia (BPH).

Methods: Sixteen Beagle dogs with BPH were randomly assigned into 4 groups $(n=4)$ : control group (without treatment), G1 group (injection with $2 \mathrm{~mL}$ of microbubble contrast agent); G2 group (21 kHz ultrasound); and G3 group (injection with $2 \mathrm{~mL}$ of microbubble contrast agent $+21 \mathrm{kHz}$ ultrasound). The histopathological damage to prostate cells was assessed via transmission electron microscopy and optical microscopy. The protein expressions of prostate-specific antigen (PSA), inducible nitric oxide synthase (iNOS), superoxide dismutase (SOD) of vessels were detected by enzyme-linked immunosorbent assay (ELISA).

Results: Histopathologically, the prostate cells exhibited nuclear chromatin contraction, mitochondrial swelling, degranulation of rough endoplasmic reticulum, basement membrane rupture and cell apoptosis in the G2 and G3 groups; it was especially obvious in the G3 group, while no changes were observed in the control and $\mathrm{G} 1$ groups. Although prostate volume using imaging was not significantly changed in all groups after treatment, PSA was significantly reduced in the $\mathrm{G} 2$ and $\mathrm{G} 3$ groups, and especially obvious in the G3 group $(p<0.05)$. The iNOS and SOD, which are important oxidative stress factors, significantly increased after treatment in the G2 and G3 groups, but not in the control and G1 groups $(p<0.05)$. Conclusions: Low-frequency ultrasound is effective in treating $\mathrm{BPH}$; low-frequency ultrasound combined with microbubbles improves the treatment efficacy.

\section{Introduction}

Benign prostatic hyperplasia $(\mathrm{BPH})$ is a non-malignant enlargement of the prostate, ranking among the 10 most common diseases in aging men. ${ }^{1} \mathrm{BPH}$ is characterized by smooth muscle and epithelial proliferation primarily within the prostatic transition zone, which results in a variety of problems for patients. The most frequent problem is lower urinary tract symptoms (LUTS) ${ }^{2}$ and $80 \%$ of men in their 70 s suffer from BPH-related LUTS., ${ }^{3,4}$

Currently, the management of BPH involves non-surgical and surgical techniques. Alpha-blockers and 5-alpha reductase inhibitors are common medical options due to their excellent efficacy and convenience of administering without severe adverse effects. ${ }^{5,6}$ Although transurethral resection of the prostate (TURP) is the gold standard for $\mathrm{BPH}$ related LUTS, it has disadvantages. Its potential disadvantages include significant blood loss and operative hyponatraemia, need for hospitalization and possible prolonged catheterization, and low, but real, risks of urinary incontinence, erectile dysfunction, bladder neck contractures, and urethral stricture disease. ${ }^{7}$ Laser surgery as a substitute has also been attempted and has a satisfactory short-term efficacy. ${ }^{8-10}$ However, elderly and more infirm patients are least likely to undergo it; this raises concern about the underutilization of the technique in this population. ${ }^{11}$ Therefore, a more effective, safe, and easy treatment strategy for BPH is urgently needed.

Ultrasound is generally used for diagnosis, ${ }^{12,13}$ and it is also being developed as a therapeutic tool related to the direct mechanical energy effects of low-frequency ultrasound. A microbubble ultrasound contrast agent is widely used as contrast media in ultrasonography, yet it is acts as a cavitation nuclei or enhancer. Low-frequency ultrasound and microbubble have been shown to accelerate thrombolysis in vitro and in vivo. ${ }^{14,15}$ In addition, low frequency ultrasound has also been effective for suppressing tumour proliferation and for promoting tumour apoptosis. ${ }^{16-18}$ However, there is currently little knowledge on the role of microbubble-mediated ultrasound in treatment of $\mathrm{BPH}$. The present study was designed, in a canine model, to demonstrate the effect of low-frequency ultrasound combined with microbubbles on $\mathrm{BPH}$. 


\section{Methods}

\section{Ultrasound system and microbubble}

The FS-450 low-frequency ultrasonic processing system (Shanghai Institute of Ultrasound in Medicine, Shanghai, China) was used in all experiments. This device is equipped with a built-in digital timer, intensity regulator and duty factor controller; settings were as follows: duty cycle, 10\%-90\%; probe frequency, $21 \mathrm{kHz}$. A sulfur hexafluoride $\left(\mathrm{SF}_{6}\right)$-filled microbubble ultrasound contrast agent (Sonovue, Bracco SpA, Milan, Italy) was used in this study, which consisted of $59 \mathrm{mg}$ of $\mathrm{SF}_{6}$ gas and $25 \mathrm{mg}$ of freeze-dried white powder. After adding $5 \mathrm{~mL}$ of $0.9 \%$ saline into the vial and shaking for several seconds, phospholipid-stabilized microbubbles filled with $\mathrm{SF}_{6}$ with a diameter of $<8 \mu \mathrm{m}$ (mean $2.5 \mu \mathrm{m}$ ) were generated at a concentration of $(2-5) \times 10^{8}$ microbubbles $/ \mathrm{mL} .{ }^{19}$ MyLab 90 ultrasound imaging system equipped with TRT33 Bi-plane electronic linear and microconvex array and transrectal transducer (4-13 MHz) (Esaote, Genoa-Florence, Italy) was used to measure the prostate diameter and volume, and the system equipped with CA431 electronic curvex array transducer $(3.5-5 \mathrm{MHz})$ was used to identify the position for displaying the prostate image.

\section{Animals and grouping}

We used 16 7-year-old male Beagle dogs diagnosed with hyperplasia nodule in the prostate by ultrasound, each weighing 17.5 to $25.6 \mathrm{~kg}$, in this study. All procedures were done in accordance with guidelines of the Chinese Council on Animal Care. Protocols were approved by the local experimental ethics committee. All animals were intravenously anesthetized with $35 \mathrm{mg} / \mathrm{kg}$ pentobarbital sodium. The lower abdomen and suprapubic region were shaved and the penis was retracted laterally to the right to provide clear acoustic access through the abdominal wall to the prostate. Transrectal ultrasound imaging and volume measurement (ellipsoid approximation) was performed using the ultrasound imaging system. The lowfrequency probe was placed on lower abdomen skin where prostate image could be displayed by the $3.5 \mathrm{MHz}$ convex array transducer near the root of the penis.

The animals were randomly divided into 4 groups: G1, G2, G3 and control; there were 4 dogs in each group. Each animal in the $\mathrm{G} 1$ group was injected with $2 \mathrm{~mL}$ of microbubble contrast agent without exposure to ultrasound; each animal in the G2 group was exposed to $21 \mathrm{kHz}$ ultrasound without microbubble contrast agent injection; each animal in the G3 group was injected with $2 \mathrm{~mL}$ of microbubble contrast agent combined with exposure to $21 \mathrm{kHz}$ ultrasound; while the other animals without ultrasound exposure and microbubble therapy served as controls.

\section{Ultrasound exposure}

The $21-\mathrm{kHz}$ transducer with $20 \mathrm{~mm}$ in diameter was positioned on the lower abdomen skin near the penis. The cold acoustic coupling gel was placed between the skin and the probe surface to prevent reflections and standing waves. The peak acoustic amplitude in degassed water was measured using a calibrated poly-(vinylidene difluoride-trifluoroethylene) needle-type hydrophone (Toray Techno Co., Ltd., Japan), $0.5 \mathrm{~mm}$ in diameter, connected to a personal or compatible computer and a digitizing oscilloscope (TDS3034, Tektronix Japan, Ltd., Japan). The spatial-average temporal average intensity (ISATA) was $318 \mathrm{~mW} / \mathrm{cm}^{2}$ and the peak acoustic pressure was $0.95 \mathrm{MPa}$, respectively. The duty ratio was $67 \%$ (the length of pulse was $20 \mathrm{~s}$ and the duty of pulse was $30 \mathrm{~s})$. The ultrasound exposure was repeated for 3 times in 3 days with a 1-day interval, of 30 minutes each time.

\section{Detection of blood samples}

Blood samples $(4 \mathrm{~mL})$ were collected from the femoral vein before treatment and after the last ultrasound exposure. The prostate-specific antigen (PSA) was detected using double antibody sandwich immunoassay, and inducible nitric oxide synthase (iNOS) and super oxidase dimutase (SOD) were measured using enzyme-linked immunosorbent assay (ELISA).

Following the last ultrasound exposure treatment, all animals were sacrificed immediately, and the prostate was surgically removed. The prostate harvested was fixed in formalin for 1 week, and then cut into pieces (measuring $10 \times 10 \times 10 \mathrm{~mm}^{3}$ ), which were subsequently dehydrated, paraffin embedded, cut into sections of $5-\mu \mathrm{m}$ in thickness with a microtome and stained with hematoxylin and eosin (H\&E). All sections were scanned with a flatbed scanner with resolution of $2400 \times 2400$ dots per inch (dpi).

\section{TUNEL}

Terminal deoxynucleotidyl transferase-mediated dUTP nick end-labeling assay (TUNEL) was performed with a TACS 2 TdT-DAB kit (Trevigen, Gaithersburg, MD) following the manufacturer's instructions. Briefly, after deparaffinization and hydration, sections were digested with proteinase $\mathrm{K}$ at a concentration of $20 \mathrm{~g} / \mathrm{mL}$ for 15 minutes. Endogenous peroxidase activity was quenched with $2 \% \mathrm{H}_{2} \mathrm{O}_{2}$ for 5 minutes. The slides were immersed in terminal deoxynucleotidyl transferase (TdT) buffer. After, TdT, $1 \mathrm{mmol} / \mathrm{L} \mathrm{Mn}^{2+}$, and biotinylated deoxynucleoside triphosphates (dNTPs) in TdT buffer were added to cover the sections and incubated in a humid atmosphere at $37^{\circ} \mathrm{C}$ for 60 minutes. The slides were washed with phosphate buffered saline (PBS) and incubated with streptavidin-horseradish peroxidase (HRP) for $10 \mathrm{~min}$ - 
utes. After being rinsed with PBS, the slides were immersed in a diaminobenzidine (DAB) solution. All slides were counterstained with $1 \%$ methyl green for 3 minutes.

\section{Statistical analysis}

All data were expressed as mean \pm standard deviation (SD), and all statistical analyses were performed using the statistical software SPSS version 17.0 (SPSS Inc., Chicago, IL). The statistical significance before and after treatment was tested using paired Student's $t$ test, and analysis of variance (ANOVA) with Greenhouse-Geisser correction was used to compare between groups. A $p<0.05$ was considered statistically significant.

Our experiments were performed with the understanding and consent of each subject, with the approval of China ethics committee.

\section{Results}

\section{Changes of prostate volume}

The mean volumes of the dog prostate in the 4 groups (control, G1, G2 and G3) were $17.16 \pm 2.07 ; 17.93 \pm 2.76$; $16.76 \pm 2.93$; and $22.62 \pm 8.89$ before treatment, respectively; after treatment the volumes were $17.26 \pm 2.19$; $17.52 \pm 2.54 ; 16.83 \pm 3.00$; and $22.38 \pm 8.93$ after treatment, respectively. No significant difference was observed in the prostate volume before and after treatment in all 4 groups (all $p>0.05$ ) (Table 1 ).

\section{Changes of PSA, iNOS and SOD levels}

We tallied the PSA, iNOS and SOD levels in the blood of dogs from the 4 groups before and after treatment (Table 2). No significant differences in the PSA, iNOS and SOD levels were observed in the control and G1 groups before and after treatment (all $p>0.05$ ), while the PSA level was significantly reduced, iNOS and SOD levels were significantly increased in the G2 and G3 groups after treatment compared with pre-treatment (all $p<0.05$ ). Apart from the lack of statistical difference in blood PSA, iNOS, and SOD level after treatment between the control and G1 groups

\begin{tabular}{|c|c|c|c|}
\hline Group & $\begin{array}{c}\text { Prostate volume } \\
\text { pre-treatment } \\
\text { (mean } \pm \mathrm{SD}, \mathrm{mm}^{3} \text { ) }\end{array}$ & $\begin{array}{c}\text { Prostate volume } \\
\text { post-treatment } \\
\left(\text { mean } \pm \mathrm{SD}, \mathrm{mm}^{3}\right)\end{array}$ & $p$ value \\
\hline Control & $22.62 \pm 8.89$ & $22.38 \pm 8.93$ & 0.488 \\
\hline G1 & $17.93 \pm 2.76$ & $17.52 \pm 2.54$ & 0.115 \\
\hline $\mathrm{G} 2$ & $16.76 \pm 2.93$ & $16.83 \pm 3.00$ & 0.817 \\
\hline G3 & $22.62 \pm 8.89$ & $22.38 \pm 8.93$ & 0.245 \\
\hline
\end{tabular}

$(p>0.05)$, there were significant differences between the G2 group and the control group, G3 group and the control groups, the $\mathrm{G} 2$ and the $\mathrm{G} 1$ group, the $\mathrm{G} 3$ and the $\mathrm{G} 1$ group (both $p<0.05$ ); a statistically significant difference was also observed between the G2 and G3 groups $(p<0.05)$.

\section{Histopathological analysis}

Under a transmission electron microscope, the prostate cells from the dogs in the G3 group exhibited nuclear chromatin contraction and margination, nuclear shrinkage (Fig. 1, part A), mitochondrial swelling, degranulation of rough endoplasmic reticulum (Fig. 1, part B), basement membrane rupture (Fig. 1, parts $C$ and D) and vacuolization. All of these changes were observed in the G2 group, but the degree was minor than in the changes in the G3 group.

Under an optical microscope, we observed infiltration of a large number of eosinophil granulocytes (Fig. 2, part A), tissue congestion, prostatic vesicle collapse (Fig. 2, part B), microthrombus formation and presence apoptotic body in the G2 and G3 groups, and more apoptotic bodies were found in the G3 group than in the G2 group. However, none of these changes were shown in the control and G1 groups.

\section{Apoptosis of prostate cells}

The TUNEL assay was performed to detect apoptosis of prostate cells. TUNEL positive cells were detected in the G2 and G3 groups, and more apoptosis was observed in the G3 group (Fig. 3, part D) than in the G2 group (Fig. 3, part C), while no positive cells were detected in the G1 (Fig. 3, part B) and control groups (Fig. 3, part A).

\begin{tabular}{|c|c|c|c|c|c|c|}
\hline Group & $\begin{array}{l}\text { PSA level pre- } \\
\text { treatment }\end{array}$ & $\begin{array}{l}\text { PSA level post- } \\
\text { treatment }\end{array}$ & $\begin{array}{c}\text { iNOS level pre- } \\
\text { treatment }\end{array}$ & $\begin{array}{l}\text { iNOS level post- } \\
\text { treatment }\end{array}$ & $\begin{array}{l}\text { SOD level pre- } \\
\text { treatment }\end{array}$ & $\begin{array}{l}\text { SOD level post- } \\
\text { treatment }\end{array}$ \\
\hline Control & $1486.74 \pm 47.99$ & $1497.05 \pm 57.23$ & $38.71 \pm 0.87$ & $37.64 \pm 2.12$ & $139.83 \pm 6.12$ & $140.63 \pm 3.97$ \\
\hline $\mathrm{G} 1$ & $1492.30 \pm 53.34$ & $1509.35 \pm 54.95$ & $38.89 \pm 0.69$ & $39.04 \pm 0.27$ & $140.20 \pm 3.50$ & $139.95 \pm 1.16$ \\
\hline G2 & $1697.04 \pm 148.36$ & $875.83 \pm 30.75$ & $40.37 \pm 1.75$ & $50.21 \pm 1.73$ & $164.51 \pm 2.70$ & $201.48 \pm 5.39$ \\
\hline G3 & $1699.39 \pm 129.71$ & $708.49 \pm 20.62$ & $37.26 \pm 4.08$ & $56.91 \pm 3.21$ & $164.87 \pm 648$ & $220.93 \pm 10.55$ \\
\hline
\end{tabular}

SD: standard deviation; G: group. 


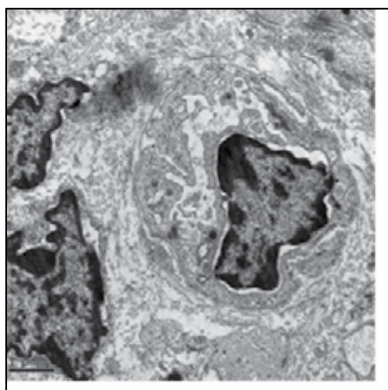

A

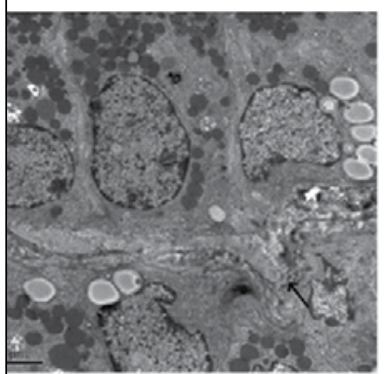

C

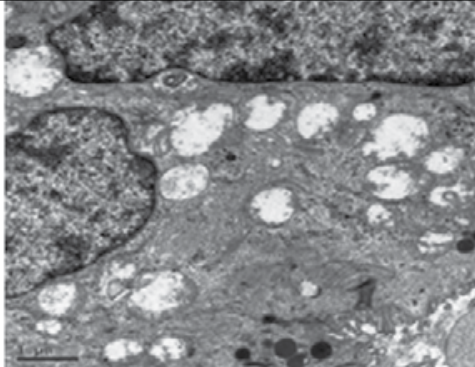

B

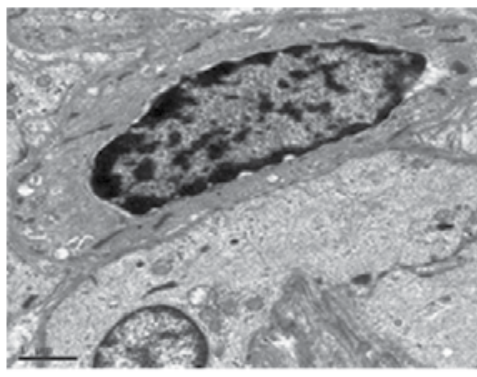

$\mathrm{D}$
Fig. 1. Transmission electron microscope analysis of the prostate cells. A: The prostate cells from the dogs in the G3 group exhibit nuclear shrinkage, nuclear chromatin contraction near the nuclear membrane and margination (arrow, $\times 10000)$. B: Mitochondrial swelling and degranulation of rough endoplasmic reticulum in the prostate cells from the dogs in the G3 group (arrow, $\times 10000)$. C: Basement membrane rupture in the prostate of the dogs in the $\mathrm{G} 3$ group (arrow, $\times 5000)$. D: Normal basement membrane $(\times 10000)$.

\section{Discussion}

Several studies have investigated the targeted treatment using low-frequency ultrasound. ${ }^{17}$ Microbubbles are cavitated by ultrasound, leading to permeabilization of prostatic capillary or small vessels followed by inducing a serial of pathological alterations such as thrombosis, micro-circulation blockage, prostatic necrosis and atrophia. Theoretically, microbubble-mediated ultrasound cavitation may be effective to treat $\mathrm{BPH} .{ }^{20}$ In the present study, an in vivo experiment was designed to further confirm our hypothesis. As expected, low-frequency ultrasound in the presence of microbubbles significantly increased the pathological damages of prostate tissues, such as nuclear chromatin condensation, swelling of mitochondria, rough endoplasmic reticulum degranulation, basement membrane rupture and cell apoptosis, compared with other groups.

Although the pathogenesis of BPH is complex, it is reported that both tissue damage and oxidative stress may lead to compensatory cellular proliferation with resulting hyperplastic growth. ${ }^{21}$ The cause of enhanced oxidative stress could be overproduction of free radicals or decrease in the activities of free radical scavenging enzymes like SOD. ${ }^{22}$ Peroxidation-antioxidant balance may be used to treat $\mathrm{BPH}$ to alter the overproduction of free radicals. In the present

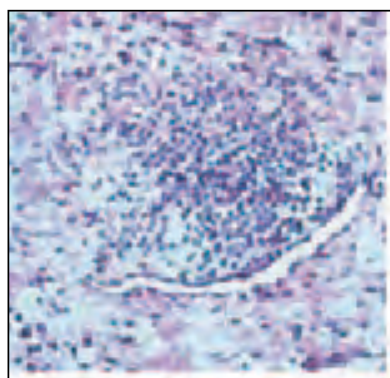

A

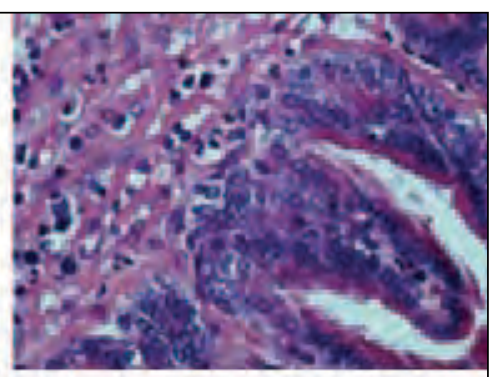

B
Fig. 2. Hematoxylin and eosin staining of the prostate cells. A: Infiltration of a large number of eosinophil granulocytes in the prostate of the dogs in the G3 group (arrow, $\times 200$ ). B: Increased prostatic vesicle nuclei and prostatic vesicle collapse in the $\mathrm{G} 3$ group (arrow, $\times 400)$.

study, the SOD significantly increased in the G2 and G3 groups after treatment; this was especially obvious in the G3 group. No significant difference was observed in the G1 and control groups compared with pre-treatment. This suggests that treatment with low-frequency ultrasound and microbubbles significantly improves $\mathrm{BPH}$.

Recently, increasing evidence indicates that nitric oxide is involved in modulating the prostatic smooth muscle relaxation; it is also involved in the control of the urethral outlet activity and in the nonadrenergic, non-cholinergic (NANC)-mediated cascades that control lower urinary tract storage and emptying. ${ }^{23}$ This suggests that an increase in the nitric oxide activity and/or its availability may alleviate $\mathrm{BPH} .{ }^{24-26}$ Both macrophages and neutrophils are the source
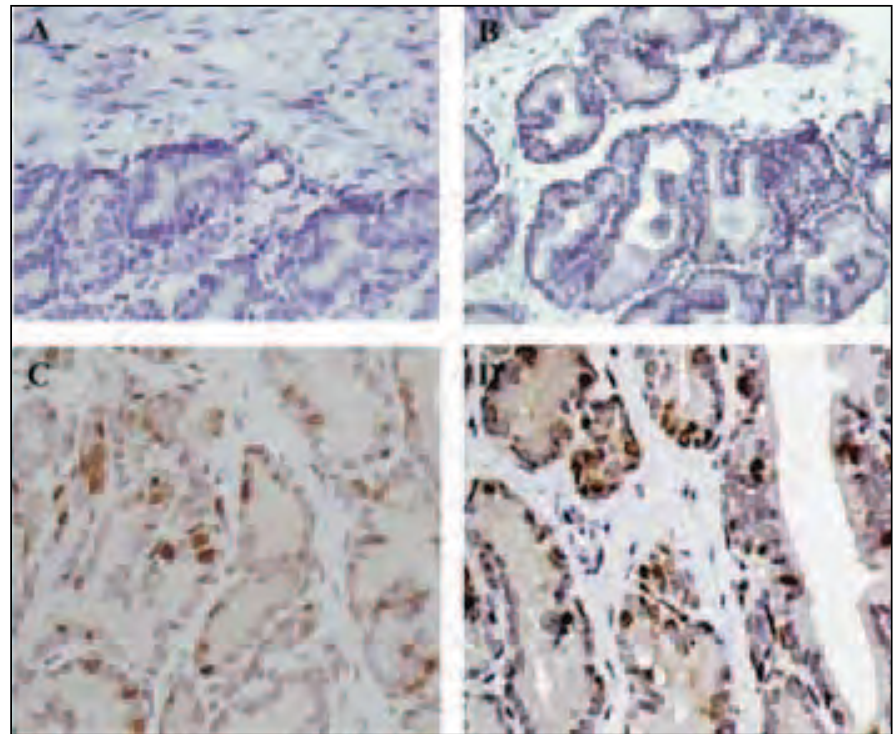

Fig. 3. Terminal uridine deoxynucleotidyl transferase nick end labelling (TUNEL) assay was performed to detect apoptosis of prostate cells. A: Control group, ( $\times 400)$; B: Sonovue group (G1, $\times 400)$; C: $21 \mathrm{kHz}$ group (G2, $\times 400)$; D: $21 \mathrm{kHz}$ +sonovuue $(\mathrm{G} 3, \times 400)$. TUNEL positive cells were detected in the $\mathrm{G} 2$ and $\mathrm{G} 3$ groups (arrow), and more apoptosis was observed in the $\mathrm{G} 3$ group than that in the $\mathrm{G} 2$ group (arrow), while no positive cells were detected in the $\mathrm{G} 1$ and control groups. 
of inducible nitric oxide synthase (iNOS) which can catalyze nitric oxide generation in a reaction where the amino acid $\mathrm{L}$-arginine is converted into $\mathrm{L}$-citrulline. ${ }^{27}$ Thus, iNOS is shown to be up-regulated after BPH treatment. As expected, our results also showed that iNOS was also significantly increased after treatment in the G2 and G3 groups. In addition, it is also suggested that high expression of iNOS in $\mathrm{BPH}$ may promote nitric oxide release, exert cytotoxic and cytostatic effects on BPH cells, and induce cells apoptosis, necrosis or DNA injury. ${ }^{28}$ This was also in line with our results of the TUNEL assay.

Furthermore, prostate volume predicts treatment outcome of $\mathrm{BPH}$, which was assessed by either transrectal ultrasound or magnetic resonance imaging and baseline serum PSA. ${ }^{29,30}$ A recent study also demonstrated that serum PSA is a stronger predictor of growth of the prostate in placebo-treated patients than baseline prostate volume. ${ }^{31}$ As expected, our results also demonstrated that there were no significant differences in prostate volume between pre-treatment and post-treatment in all groups by using imaging. However, the PSA was shown to be significantly reduced in the G2 and G3 groups; it was especially obvious in the G3 group. This result concurs with previous studies. ${ }^{32}$

\section{Conclusion}

Low-frequency ultrasound is effective in treating $\mathrm{BPH}$; combined low-frequency ultrasound and microbubbles further improves the treatment efficacy by increasing iNOS and SOD, but decreasing PSA. However, there are still some limitations in this study. We applied low-frequency ultrasound $(20 \mathrm{kHz})$ to explore its treatment effect for $\mathrm{BPH}$. However, whether low frequency ultrasound $(1 \mathrm{MHz})$ is also effective or whether the therapeutic effect of $20 \mathrm{kHz}$ ultrasound is superior to $1 \mathrm{MHz}$ remains unclear. In addition, how the apoptosis factors (such as caspase-3, Bcl-2 and survivin) change remains under-investigated. Further studies are still needed.

Acknowledgements: This study was supported by the National Natural Science Fund (grant No. 81271597) and the Natural Science Foundation of Shanghai (grant No. 10JC1412600). Yang Shao-ling and Tang Ke-qiang are co-first authors.

Competing interests: Dr. Yang Shao-ling, Dr. Tang Ke-qiang, Dr. Bai Wen-kun, Dr. Shen, Dr. Zhao Yi-wen, Dr. Lin Yan-duan, Dr. Nan Shu-liang, and Dr. Hu Bing all declare no competing financial or personal interests.

This paper has been peer-reviewed.

\section{References}

1. Issa MM, Regan TS. Medical Therapy for Benign Prostatic Hyperplasia-Present and Future Impact-Page 3. Am J Manag Care 2007;13:S4-S9.

2. Minutoli L, Bitto A, Squadrito F, et al. Serenoa Repens, Lycopene and Selenium: A Triple Therapeutic Approach to Manage Benign Prostatic Hyperplasia. Curr Med Chem 2013;20:1306-12.

3. Roehrborn CG. Male lower urinary tract symptoms (LUTS) and benign prostatic hyperplasia (BPH). Med Clin North Am 2011;95:87-100. http://dx.doi.org/10.1016/i.mena.2010.08.013

4. McNicholas T, Kirby R. Benign prostatic hyperplasia and male lower urinary tract symptoms (LUTS). Clin Evid (Online) 2011;2011.

5. Lepor H. Alpha blockers for the treatment of benign prostatic hyperplasia. Rev Urol 2007;9:181-90.

6. Barkin J. Review of dutasteride/tamsulosin fixed-dose combination for the treatment of benign prostatic hyperplasia: efficacy, safety, and patient acceptability. Patient Prefer Adherence 2011;5:483-90.

7. Zhang X, Geng J, Zheng J, et al. Photoselective Vaporization Versus Transurethral Resection of the Prostate for Benign Prostatic Hyperplasia: A Meta-Analysis. J Endourol 2012;26:1109-17. http://dx.doi. org/10.1089/end.2012.0136

8. Gravas $\mathrm{S}$, Bachmann $\mathrm{A}$, Reich 0 , et al. Critical review of lasers in benign prostatic hyperplasia (BPH). BJU Int 2011;107:1030-43. http://dx.doi.org/10.1111/j.1464-410X.2010.09954.x

9. Bachmann A, Woo HH, Wyler S. Laser prostatectomy of lower urinary tract symptoms due to benign prostate enlargement: a critical review of evidence. Curr Opin Urol 2012;22:22-33. http://dx.doi. org/10.1097/MOU.0b013e32834dd0ed

10. Rassweiler J, Teber D, Kuntz R, et al. Complications of transurethral resection of the prostate (TURP) incidence, management, and prevention. Eur Urol 2006;50:969-80. http://dx.doi.org/10.1016/i. eururo.2005.12.042

11. Schroeck FR, Hollingsworth JM, Kaufman SR, et al. Population Based Trends in the Surgical Treatment of Benign Pr ostatic Hyperplasia. J Urol 2012;188:1837-41. http://dx.doi.org/10.1016/i. juro.2012.07.049

12. Veselka J, Čadová P, Adla T, et al. Dual-source computed tomography angiography and intravascular ultrasound assessment of restenosis in patients after coronary stenting for bifurcation left main stenosis: a pilot study. Arch Med Sci 2012;8:455-61. http://dx.doi.org/10.5114/aoms.2012.29220

13. Xue N, Huang $P$, Aronow WS, et al. Predicting lymph node status in patients with early gastric carcinoma using double contrast-enhanced ultrasonography. Arch Med Sci 2011;7:457-64. http://dx.doi. org/10.5114/aoms.2011.23412

14. Xie F, Lof J, Everbach C, et al. Treatment of acute intravascular thrombi with diagnostic ultrasound and intravenous microbubbles. JACC Cardiovasc Imaging 2009;2:511-8. http://dx.doi.org/10.1016/i. jicmg.2009.02.002

15. Wang B, Wang L, Zhou X-B, et al. Thrombolysis effect of a novel targeted microbubble with low-frequency ultrasound in vivo. Thromb Haemost 2008; 100:356-61.

16. Goertz DE, Karshafian R, Hynynen K. Investigating the effects of pulsed low intensity ultrasound and microbubbles in mouse tumors. In: Ultrasonics Symposium (IUS); 2009:89-92.

17. Shen Z-Y, Hu B. Low-frequency low-intensity ultrasound with contrast agent for the treatment of subcutaneous tumors in mice. Scientific Research and Essays 2011;6:5579-85.

18. Zhang Z, Chen J, Chen L, et al. Low frequency and intensity ultrasound induces apoptosis of brain glioma in rats mediated by caspase-3, Bcl-2, and survivin. Brain Res 2012;1473:25-34. http://dx.doi. org/10.1016/i.brainres.2012.06.047. Epub 2012 Jul 20.

19. Cozar J, Solsona E, Brenes F, et al. Clinical management of patient with benign prostatic hyperplasia in Spain [in Spanish]. Actas Urol 2011;35:580-8. http://dx.doi.org/10.1016/i.acuro.2011.07.008. Epub 2011 Sep 28.

20. Li T, Liu Z. Ablation of benign prostatic hyperplasia using microbubble-mediated ultrasound cavitation. Med Hypotheses 2010;74:679-80. http://dx.doi.org/10.1016/i.mehy.2009.10.052

21. Chughtai B, Lee R, Te A, et al. Role of inflammation in benign prostatic hyperplasia. Rev Urol 2011;13:14750.

22. Ahmad M, Suhail N, Mansoor T, et al. Evaluation of Oxidative Stress and DNA Damage in Benign Prostatic Hyperplasia Patients and Comparison with Controls. Indian J Clin Biochem 2012;27:385-8. http://dx.doi. org/10.1007/s12291-012-0229-4

23. Yoshida $M$, Akaike $T$, Inadome $A$, et al. The possible effect of nitric oxide on relaxation and noradrenaline release in the isolated rabbit urethra. Eur J Pharmacol 1998;357:213-9. http://dx.doi.org/10.1016/ S0014-2999(98)00566-4

24. Kang KK, Kim JM, Yu JY, et al. Effects of phosphodiesterase type 5 inhibitor on the contractility of prostate tissues and urethral pressure responses in a rat model of benign prostate hyperplasia. Int J Urol 2007;14:946-51. http://dx.doi.org/10.1111/j.1442-2042.2007.01874.x 
Shao-ling et al.

25. Kedia GT, Ückert $S$, Jonas $U$, et al. The nitric oxide pathway in the human prostate: clinical implications in men with lower urinary tract symptoms. World J Urol 2008;26:603-9. http://dx.doi.org/10.1007/ s00345-008-0303-y

26. Yoo TK, Cho HJ. Benign prostatic hyperplasia: from bench to clinic. Korean J Urol 2012;53:139-48.

27. Albina JE. On the expression of nitric oxide synthase by human macrophages. Why no NO? J Leukoc Biol 1995;58:643-9.

28. Skorpikova J, Dolnikova M, Hrazdira I, et al. Changes in microtubules and microfilaments due to a combined effect of ultrasound and cytostatics in HeLa cells. Folia Biol (Praha) 2001;47:143-7.

29. Roehrborn CG, Boyle P, Gould AL, et al. Serum prostate-specific antigen as a predictor of prostate volume in men with benign prostatic hyperplasia. Urology 1999;53:581-9. http://dx.doi.org/10.1016/ S0090-4295(98)00655-4

30. Roehrborn CG, Boyle P, Bergner D, et al. Serum prostate-specific antigen and prostate volume predict long-term changes in symptoms and flow rate: results of a four-year, randomized trial comparing finasteride versus placebo. Urology 1999:54:662-9. http://dx.doi.org/10.1016/S0090-4295(99)00232-0
31. Roehrborn CG, MCConnell J, Bonilla J, et al. Serum prostate specific antigen is a strong predictor of future prostate growth in men with benign prostatic hyperplasia. J Urol 2000;163:13-20. http://dx.doi. org/10.1016/50022-5347(05)67962-1

32. Song L, Zhu Y, Han P, et al. A Retrospective Study: Correlation of Histologic Inflammation in Biopsy Specimens of Chinese Men Undergoing Surgery for Benign Prostatic Hyperplasia With Serum Prostatespecific Antigen. Urology 2011;77:688-92. http://dx.doi.org/10.1016/i.urology.2010.07.493

Correspondence: Dr. Bing Hu, Department of Ultrasound in Medicine, Shanghai Jiao Tong University, Affiliated 6th People's Hospital, Shanghai Institute of Ultrasound in Medicine, Yishan Road, 600, Shanghai 200233, P.R. China; binghu_stephen@163.com 